\title{
Analysis of Potential Drug Interactions in Brazilian Mental Health Services: Prevalence and Associated Factors
}

\author{
Sarah Nascimento Silva*, Marina Guimarães Lima, Cristina Mariano Ruas \\ Department of Social Pharmacy, School of Pharmacy, Federal University of Minas Gerais, Belo Horizonte, BRAZIL.
}

\begin{abstract}
Background: In the mental health field, the psychotropic polypharmacy is frequently observed, a significant risk factor for the occurrence of drug interactions. Objective: Identify potential drug interactions on the prescriptions of mental health services users and describe the associated factors. Methods: A cross-sectional study was conducted in 11 services. Sociodemographic data and information about the use of drug were obtained through interviews of users, analysis of prescriptions and medical charts, using a semi-structured questionnaire. Potential drug interactions were identified using the Micromedex $₫$ database, the association with sociodemographic characteristics and aspects related to the medicines prescribed were analyzed using the prevalence ratio. Results: The number of medicines prescribed ranged from 0 to 9 , with an average of $3.38(S D=1.76)$ per user, the most prescribed being haloperidol (12.3\%), clonazepam (8.2\%) and biperiden $(7.9 \%)$. The proportion of interactions considering the number of users interviewed was $35.1 \%$. The most frequent between potential interactions were haloperidol and fluoxetine (9.1\%); haloperidol and carbamazepine (8.8\%); and carbamazepine and chlorpromazine (5.9\%). The highest prevalence ratio (PR) for the occurrence of potential interactions was
\end{abstract}

in women ( $P R=1.36 ; 95 \% \mathrm{Cl} 1.08: 1.71)$, users who had reported improper medicine use ( $\mathrm{PR}=1.36 ; 95 \% \mathrm{Cl}: 1.07: 1.72)$ and those with more than 5 prescribed medicines ( $\mathrm{PR}=1.87 ; 95 \% \mathrm{Cl}$ : 1.49:2.33). Conclusion: Potential drug interactions were observed in more than one-third of the user's Brazilian mental health services. The profile of interactions detected could guide the pharmacotherapeutic follow-up of priority users, and help the multidisciplinary team identify signs and symptoms that can influence the treatment of users.

Key words: Drug interactions, Drug prescriptions, Psychotropic drugs, Mental health, Mental health services.

Correspondence

Sarah Nascimento Silva, Federal University of Minas Gerais, Av. Pres. Antônio Carlos, 6627 - Pampulha 31270-901. Belo Horizonte, Minas Gerais, BRAZIL.

Phone: +556134095000

Email: sarahnsilva@gmail.com

DOI: 10.5530/jyp.2018.10.52

\section{INTRODUCTION}

Drug interactions are one of the main problems related to the use of medication. ${ }^{1}$ In addition to clinically compromising the patient, interactions are also associated with increased hospitalization time and higher treatment costs. ${ }^{2}$ In the mental health field, the use of psychotropic drugs is an important therapeutic resource. Many of these drugs exhibit a narrow therapeutic index, which may be associated with more severe drug interactions. ${ }^{3}$ Furthermore, polypharmacy is frequently observed in mental health treatment ${ }^{4}$ and is a significant risk factor for the occurrence of drug interactions, ${ }^{3}$ demonstrating the need to monitor these occurrences. ${ }^{5}$

Drug interactions that involve psychotropic primarily compromise the metabolization process. Interactions may considerably raise the risk of intoxication, due to the inhibition of some cytochrome P450 isoenzymes, and exacerbate adverse reactions, ${ }^{6}$ as occurs in several antipsychotic drugs. ${ }^{1}$ Other interactions may trigger a reduction in the plasma concentration of a number of drugs, or even specific syndromes and symptoms. ${ }^{6}$ These changes in pharmacokinetic and pharmacodynamics aspects may interfere in the main results that the patient seeks to achieve, namely, symptom elimination, quality of life and disease stabilization. ${ }^{6}$

Drug interaction studies conducted at mental health outpatient facilities are scarce, ${ }^{7}$ and most are restricted to a hospital setting, ${ }^{8}$ although is an important aspect to consider in therapeutic planning and patient safety. ${ }^{7}$ Psychosocial Care Centers (CAPS - from Portuguese Centro de Atenção Psicossocial) are strategic services belonging to the Psychosocial Care Network and the Brazilian health system. ${ }^{9}$ Identifying the most frequent potential interactions may be important in subsidizing clinical practice pharmacovigilance, and planning initiatives to improve patient safety and rational drug use. The aim of the present study was to identify the most frequent potential drug interactions on the prescriptions of CAPS users.

\section{MATERIALS AND METHODS}

A cross-sectional study was conducted with the users of 11 CAPS in the Médio Paraopeba region of Minas Gerais state (MG), Brazil. Eligible CAPS were those located in medium-sized and large cities, with populations of more than 100,000 inhabitants. Participant selection considered a systematic sample of users treated at the services between August 2014 and February 2015, deemed stable by the CAPS team, that is, with no episodes and capable of understanding the researchers' questions. Excluded were users who did not understand the questions, even after the researcher's explanations, thereby characterizing some degree of mental confusion or agitation. The users or their guardians who agreed to participate in the study gave their informed consent. After the interviews were conducted, the medical prescriptions and/or medical records were analyzed.

The sociodemographic characteristics of users, prescribed drugs and reports on inappropriate use of medicines use were described using measures of central tendency, dispersion and proportion. The units of analysis were the patient, the medicine and the drug interaction. The medicines were classified according to the Anatomical Therapeutic

This is an open access article distributed under the terms of the Creative Commons Attribution-NonCommercial-ShareAlike 4.0 License, which allows others to remix, tweak, and build upon the work non-commercially, as long as the author is credited and the new creations are licensed under the identical terms. 
Chemical Classification System (ATC) and grouped under the third level to establish the main classes prescribed at these services. Interactions were identified using the Micromedex ${ }^{\star}$ database. Potential interactions with clinical relevance were determined, that is, those with excellent or good quality documentation and considered major, moderate or contraindicated.

The association between the occurrence of a potential drug interaction, sociodemographic characteristics and aspects related to the medicines prescribed were analyzed using the prevalence ratio, considering a 95\% confidence interval. The programs used in statistical analyses were IBM SPSS Statistics version 19.0 and Microsoft Excel.

The study was approved by the Research Ethics Committee of the Federal University of Minas Gerais under protocol number 1160959 and CAAE 26041113.6.0000.5149. Patients were invited to participate in the study by signing the Free and Informed Consent Term (TCLE) of the research, according to Brazilian ethical legislation. The responsible for the user could sign the TCLE and participate concurrently in the interview with the user.

\section{RESULTS}

A total of 467 users were interviewed. The CAPS users were mostly male $(63.17 \%)$, single $(67.75 \%)$, with a predominant age group between 25 and 59 years (65.59\%), the mean age of participants was 34 years $(\mathrm{SD}=16.54)$. Educational level was concentrated in the initial years of the graded school (51.49\%), those who worked, generally exercised occupations with low level of qualification (34.74\%).

The number of medicines prescribed at the CAPS ranges from 0 to 9 , with an average of 3.38 medicines $(\mathrm{SD}=1.76)$ per user, the most prescribed being haloperidol (12.3\%), clonazepam (8.2\%), biperiden (7.9\%), diazepam (7.3\%) and valproic acid (6.4\%) (Table 1$)$. The most frequently prescribed classes of drugs, in accordance with the third ATC classification level, were antipsychotics (37.5\%), antiepileptics (21.5\%) and antidepressants (11.9\%).

The occurrence of potential drug interactions in the 391(83.7\%) users that were prescribed 2 or more medicines was investigated and 164 (41.9\%) displayed between 1 and 8 potential interactions, with a mean value of $0.78(\mathrm{SD}=1.26)$ interactions per user. The proportion of interactions considering the number of users interviewed was $35.1 \%$. Of the 306 potential interactions identified, 155 (50.6\%) were classified as severe, $150(49.0 \%)$ moderate and only one $(0.3 \%)$ was contraindicated. With respect to the quality of the interaction documents, most were classified by the Micromedex ${ }^{\oplus}$ database as good ( $\left.\mathrm{n}=270 ; 88.7 \%\right), 24(7.8 \%)$ excellent and $12(3.9 \%)$ were not classified due to being manually extracted on the platform.

Fifty-one different combinations of potential interactions were identified in this study, the most frequent between haloperidol and fluoxetine (9.1\%); haloperidol and carbamazepine (8.8\%); and carbamazepine and chlorpromazine (5.9\%) (Table 2).The highest prevalence ratio (PR) for the occurrence of potential interactions was in women $(\mathrm{PR}=1.36 ; 95 \%$ CI 1.08:1.71), users who had reported improper medicine use ( $\mathrm{PR}=1.36$; 95\% CI 1.07:1.72), those with more than 5 prescribed medicines $(\mathrm{PR}=1.87 ; 95 \%$ CI 1.49:2.33), and individuals whose prescription contained antidepressants ( $\mathrm{PR}=1.71 ; 95 \%$ CI 1.36-2.15), antipsychotics $(\mathrm{PR}=1.72 ; 95 \%$ CI $1.17: 2.54)$ or antiepileptics ( $\mathrm{PR}=1.81 ; 95 \% \mathrm{CI}$ $1.34: 2.44)$. The economically active age group, that is, aged between 20 and 59 years, was a protective factor for the occurrence of potential drug interactions ( $\mathrm{PR}=0.50$; 95\% CI 0.32:0.78).

\section{DISCUSSION}

The prevalence of potential drug interactions in this study was $35.1 \%$, a value similar to that found in a number of general hospitals in Brazil, whose prevalence varied from $22^{3}$ to $37 \% .^{2}$ This value, however, was lower than those reported in studies conducted at basic care facilities $(48.7 \%),{ }^{10}$ even when the focus was on treating mental disorders(58.4\%). ${ }^{7}$ The prevalence of potential interactions was higher than a study with patients with schizophrenia using antipsychotics $(23 \%),{ }^{1}$ but lower compared to another study with the same population that included in their investigation besides the medicines for the treatment of mental disorders, antihypertensive drugs in use by the patients $(65 \%) .{ }^{11}$ Given the wide variation in the prevalence of interactions, even between general and psychiatric hospitals, ${ }^{8}$ results should be compared with caution, including the analysis of different CAPS modalities, since they treat populations and clinical conditions differently.

The most frequent interactions involved fluoxetine, haloperidol and carbamazepine, the last two also recurring in interactions detected at basic care facilities that treat mental disorders. ${ }^{12}$ The frequent use of haloperidol in this and other studies underscores the important role of

Table 1: Medicines more prescribed in Psychosocial Care Centers users in the Médio Paraopeba region, Minas Gerais, Brazil 2014-2015.

\begin{tabular}{ccc}
\hline Medicamento & ATC class & Prescription frequency $\mathbf{n}(\%)$ \\
\hline haloperidol & Antipsychotics (N05A) & $193(12.3)$ \\
clonazepan & Antiepileptics (N03A) & $128(8.2)$ \\
biperiden & Anticholinergic Agents (N04A) & $124(7.9)$ \\
diazepam & Anxiolytics (N05B) & $114(7.3)$ \\
valproic acid & Antiepileptics (N03A) & $101(6.4)$ \\
chlorpromazine & Antipsychotics (N05A) & $95(6.1)$ \\
levomepromazine & Antipsychotics (N05A) & $88(5.6)$ \\
carbamazepine & Antiepileptics (N03A) & $80(5.1)$ \\
fluoxetine & Antidepressants (N06A) & $69(4.4)$ \\
Vitamin B-complex & Vitamin b-complex, incl. combinations(A11E) & $63(4.0)$ \\
risperidone & Antipsychotics (N05A) & $61(3.9)$ \\
thiamine & Vitamin B1 (A11D) & $55(3.5)$ \\
lithium & Antipsychotics (N05A) & $52(3.3)$ \\
\hline
\end{tabular}

ATC: Anatomical Therapeutic Chemical Classification System. 


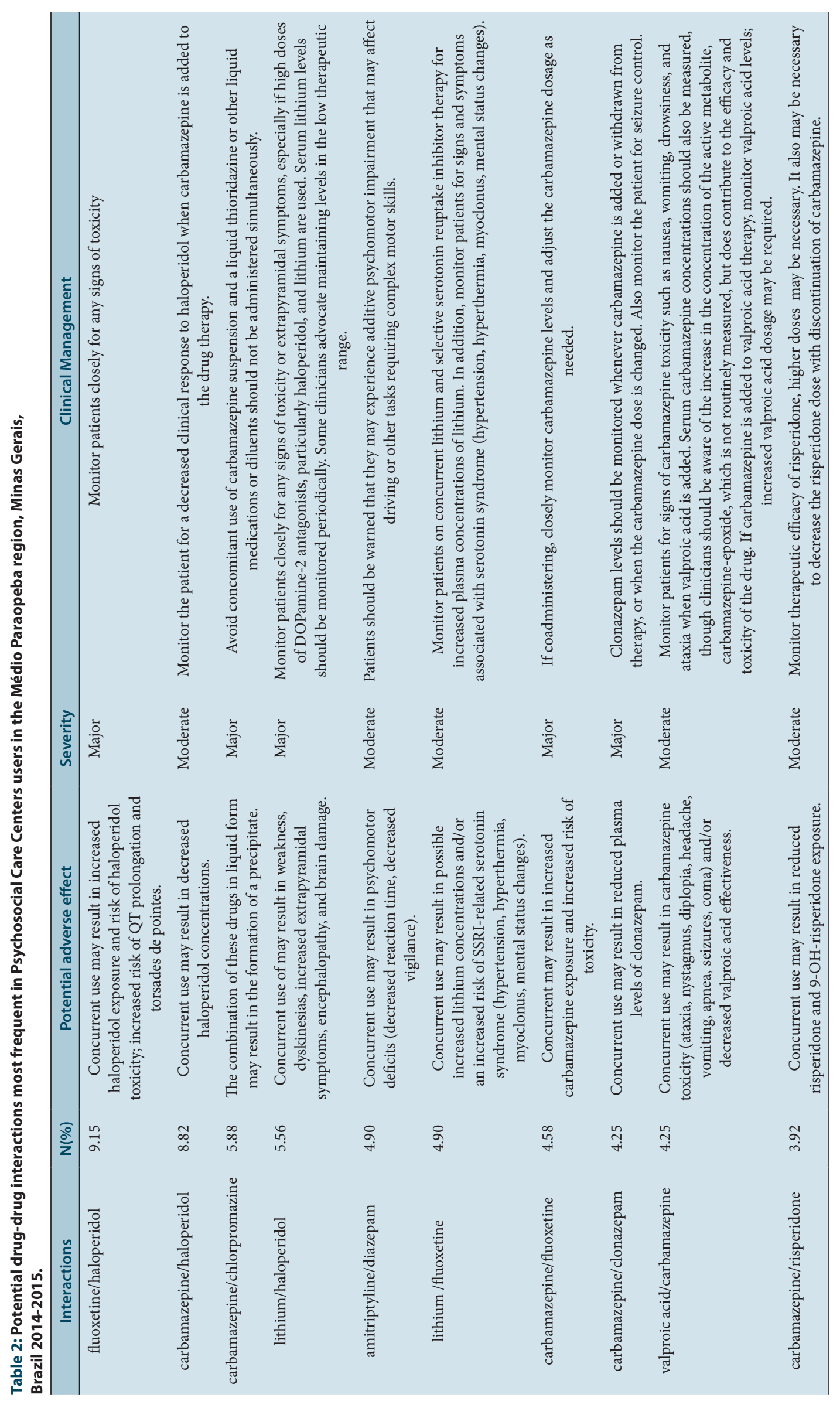


this drug in antipsychotic polytherapy, as well as concerns regarding its prescription patterns in the treatment of mental health. ${ }^{13}$ Identifying the main potential interactions at the CAPS is important in guiding the multidisciplinary team, and monitoring the signs, symptoms and toxic effects of drug interactions on priority groups of users, until their inclusion in all the services.

Analysis of potential drug interactions should be part of therapeutic planning for CAPS users, especially those at greater risk of polytherapy, ${ }^{3}$ children and the elderly. ${ }^{5}$ A study in Australia showed that the identification of drug interactions by pharmacists was one of the most common interventions in psychiatric hospitals, ${ }^{14}$ in contrast to the situation of the CAPS in this region. ${ }^{15}$ Moreover, in mental health, identifying severe interactions that pose a health risk should be only one of the aspects investigated, since establishing the clinical relevance of interactions for these users may also be crucial. Exacerbating a side effect that poses no serious health risk but affects the routine and behavior of the user, could be clinically significant and may negatively affect adherence to therapy, a frequent and relevant problem for these users. ${ }^{12}$

The clinical management to be carried out for more frequent interactions are related to the monitoring of serum levels of the drugs and mainly to the occurrence of typical symptoms of intoxication by one of the medicines. The Serotonin syndrome is a potentially lethal condition that can be caused by interactions with medications in the treatment of mental disorders and requires the attention of health professional. ${ }^{16}$ It is observed that the performance of the pharmacist in these services, informing and alerting about drug interactions is very important in the education of professionals and patients. ${ }^{17}$ The pharmacist may alert you to unexpected symptoms and adverse effects, the clinical practice may help minimize adverse drug reactions, avoiding the administration of more drugs with potential side effects. ${ }^{17}$

This is a pioneering study in reporting interactions detected in CAPS users, a recent service offered by the Brazilian health system. The results may lead to new studies and guide the prioritization of interventions in CAPS aimed at patient safety and other aspects that may interfere with the adhesion and follow-up of pharmacotherapy.

\section{Limitations of the study}

This is a cross-sectional study, so more studies are necessary for other periods to confirm the profile of potential interactions. Analysis of the potential interactions was limited to a non-random sample and was restricted to drugs prescribed by CAPS, which suggests that this value is underestimated. The potential drug interactions do not necessarily reflect the actual occurrence of interactions in clinical practice but provide important information to minimize the risk of interactions and ensure the efficacy and safety of the proposed therapy.

\section{CONCLUSION}

The result highlights the occurrence of potential drug interactions in more than one-third of the users of these services, which should be investigated in future studies, considering dosage, drug use duration, diagnosis and other factors that are important for the occurrence of high-risk drug interactions. CAPS users often undergo polytherapy and are exposed to drugs with a significant potential for interactions. The profile of interactions detected could guide the pharmacotherapeutic follow-up of priority users, and help the multidisciplinary team identify signs and symptoms that can influence the clinical status and treatment of CAPS users.

\section{CONFLICT OF INTEREST}

The authors report no conflict of interest.

\section{REFERENCES}

1. Guo JJ, Wu J, Kelton CML, Jing Y, Fan H, Keck PE, et al. Exposure to potentially dangerous drug-drug interactions involving antipsychotics. Psychiatr Serv. 2012;63(11):1080-8.

2. Moura CSD, Acúrcio FDA, Belo NDO. Drug-drug interactions associated with length of stay and cost of hospitalization. J Pharm Pharm Sci. 2009;12(3):266-72.

3. Moura CS, Ribeiro AQ, Starling SM. Avaliação de interações medicamentosas potenciais em prescrições médicas do Hospital das Clínicas da Universidade Federal de Minas Gerais (Brasil). Lat Am J Pharm. 2007;26(4):596-601.

4. Mojtabai R, Olfson M. National trends in psychotropic medication polypharmacy in office-based psychiatry. Archives of General Psychiatry. 2010;67(1):26-36.

5. Novaes PH, Da Cruz DT, Lucchetti ALG, Leite ICG, Lucchetti G. The" iatrogenic triad": polypharmacy, drug-drug interactions, and potentially inappropriate medications in older adults. Int J Clin Pharm. 2017;39(4):818-25.

6. English BA, Dortch M, Ereshefsky L, Jhee S. Clinically significant psychotropic drug-drug interactions in the primary care setting. Curr Psychiatry Rep. 2012;14(4):376-90.

7. Borges TL, Vedana KGG, Castilho ECD, Miasso Al. Factors Associated with Potential Drug-Drug Interactions in Patients Attended in Primary Health Care: A Focus on Mental Health. Issues Ment Health Nurs. 2017;38(9):769-74.

8. Sinclair LI, Davies SJC, Parton G, Potokar JP. Drug-drug interactions in general hospital and psychiatric hospital in-patients prescribed psychotropic medications. Int J Psychiatry Clin Pract. 2010;14(3):212-9.

9. Trapé TL, Campos RO. The mental health care model in Brazil: Analyses of the funding, governance processes, and mechanisms of assessment. Rev Saúde Pública. 2017;51:19.

10. Leao DFL, De Moura CS, De Medeiros DS. Avaliação de interações medicamentosas potenciais em prescrições da atenção primária de Vitória da Conquista (BA), Brasil. Ciênc Saúde Colet. 2014;19(1):311-8.

11. Ocaña-Zurita MC, Juárez-Rojop IE, Genis A, Tovilla-Zárate CA, González-CastroTB, López-Narváez ML, et al. Potential drug-drug interaction in Mexican patients with schizophrenia. Int J Psychiatry Clin Pract. 2016;20(4):249-53.

12. Miasso Al, Telles Filho PCP, Borges TL, Pereira Júnior ADC, Giacchero Vedana KG, Shasanmi R, et al. Adherence to Psychotropic Medications and Associated Factors in Primary Health Care. Issues Ment Health Nurs. 2016;37(10):775-83.

13. Armstrong KS, Temmingh $\mathrm{H}$. Prevalence of and factors associated with antipsychotic polypharmacy in patients with serious mental illness: Findings from a cross-sectional study in an upper-middle-income country. Rev Bras Psiquiatr. 2017;39(4):293-301.

14. Richardson TE, O'Reilly CL, Chen TF. Drug-related problems and the clinical role of pharmacists in inpatient mental health: An insight into practice in Australia. Int J Clin Pharm. 2014;36(5):1077-86.

15. Silva SN, Lima MG. Assistência Farmacêutica na Saúde Mental: Um diagnóstico dos Centros de Atenção Psicossocial. Ciênc Saúde Colet. 2017;22(6):2025-36.

16. Ables AZ, Nagubilli R. Prevention, recognition, and management of serotonin syndrome. Am Fam Physician. 2010;81(9):1139-42

17. Ansari JA. Drug interaction and pharmacist. J Young Pharm. 2010;2(3):326-31. 